\title{
Recent advances in functional nanostructures as cancer photothermal therapy
}

This article was published in the following Dove Press journal:

International Journal of Nanomedicine

\author{
Essraa A Hussein' \\ Moustafa M Zagho' \\ Gheyath K Nasrallah",3 \\ Ahmed A Elzatahry' \\ 'Materials Science and Technology \\ Program, College of Arts and Sciences, \\ Qatar University, Doha, Qatar; \\ ${ }^{2}$ Department of Biomedical Science, \\ College of Health Sciences, Qatar \\ University, Doha, Qatar; ${ }^{3}$ Biomedical \\ Research Center, Qatar University, \\ Doha, Qatar
}

\begin{abstract}
Being a non-invasive and relatively safe technique, photothermal therapy has attracted a lot of interest in the cancer treatment field. Recently, nanostructure technology has entered the forefront of cancer therapy owing to its ability to absorb near-infrared radiation as well as efficient light to heat conversion. In this study, key nanostructures for cancer therapy including gold nanoparticles, magnetite iron oxide nanoparticles, organic nanomaterials, and novel twodimensional nanoagents such as MXenes are discussed. Furthermore, we briefly discuss the characteristics of the nanostructures of these photothermal nanomaterial agents, while focusing on how nanostructures hold potential as cancer therapies. Finally, this review offers promising insight into new cancer therapy approaches, particularly in vivo and in vitro cancer treatments.
\end{abstract}

Keywords: photothermal therapy, nanostructures, near-infrared, plasmonic, cancer therapy

\section{Introduction}

Globally, cancer as an invasive and malignant disease is considered the second leading cause of mortality. As per the American Cancer Society, the estimated number of cancer incidence in 2017 is 1,688,780 new cancer cases and 600,920 cancer deaths in USA, which implies a potential increase of $70 \%$ over the next 2 decades. ${ }^{1,2}$ Current cancer therapies, such as surgical excision, chemotherapy, radiation therapy, or even combination therapy, have severe limitations. Surgery could result in hurt or injury in the adjacent healthy critical tissues, and both chemotherapy and radiotherapy exhibit violent side effects. ${ }^{3}$

Recently, photothermal therapy (PTT) has gained a great deal of attention due to its excellent performance in cancer treatment. PTT depends on heat generation upon exposure of tumor cells to near-infrared radiation (NIR). ${ }^{4-6}$ PTT agents absorb light energy, which causes electron excitation and subsequent non-radiative relaxation. This will lead to gain kinetic energy, which in turn results in heat generation in the medium surrounding the PTT agents. ${ }^{7-9}$ This PTT approach is considered as non-invasive thermotherapy in which the induced thermal energy develops cell membrane disruption or protein denaturation of the surrounding tumor cells. ${ }^{10}$

Coupled with that, a major breakthrough in nanotechnology has been developed resulting in the production of various nanomaterials for many applications. Due to their unique optical properties, nano-scaled materials, especially noble metal nanoparticles (NPs), are promising in the nanomedicine and biomedical fields. ${ }^{11-14}$ The noble metal NPs such as gold (Au) and silver (Ag) have a unique photo-physical phenomenon, called localized surface plasmon resonance (LSPR), which enhances their photoabsorption. ${ }^{15-18}$ Consequently, they are considered as PTT because of their ability to stimulate hyperthermia in the cancerous cells, when exposed to NIR. ${ }^{19-21}$ This phenomenon is due to the special optical properties of noble metal NPs, which are able
Correspondence: Ahmed A Elzatahry Materials Science and Technology Program, College of Arts and Sciences, Qatar University, PO Box 27I3,

Doha, Qatar

Email aelzatahry@qu.edu.qa 

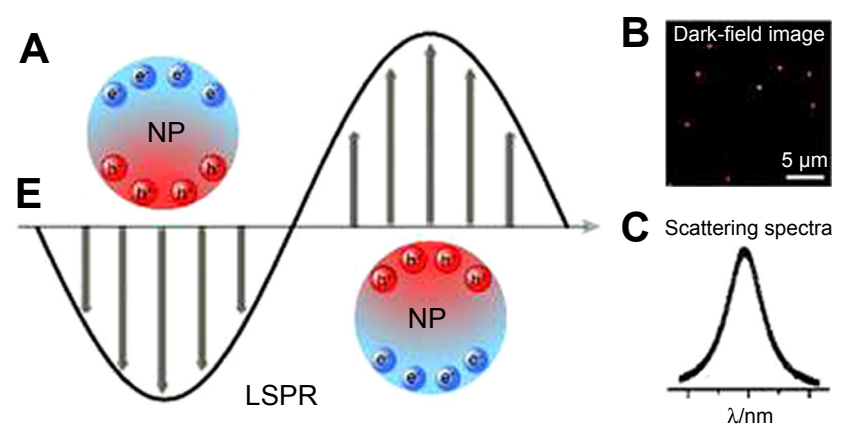

Figure I (A) Schematic description of localized surface plasmon resonance in metal NPs. (B) Dark-field image of NPs. (C) Scattering spectrum of a single NP.

Notes: Reproduced from Xie T, Jing C, Long YT. Single plasmonic nanoparticles as ultrasensitive sensors. Analyst. 20I7;142(3):409-420, ${ }^{25}$ http://pubs.rsc.org/en/ Content/ArticleLanding/20 I7/AN/C6AN0 I852A\#!divAbstract, with permission of The Royal Society of Chemistry.

Abbreviations: LSPR, localized surface plasmon resonance; NP, nanoparticle.

to absorb the light energy in NIR region (ie, 650-900 nm). Then, the absorbed light energy is converted to heat leading to tumor cell damage and tumor tissue necrosis. ${ }^{3}$

To illustrate, the surface plasmon resonance (SRP) effect means that the metal-free electrons that exist in the conduction band oscillate around the particle surface when exposed to the electromagnetic field of light. Subsequently, this oscillation leads to charge separation and formation of dipole oscillation (Figure 1). At a certain frequency, the oscillation will reach its maximum which is known as SPR, which supports the light absorption of the NPs, especially the noble metals (ie, Au and Ag). ${ }^{22-25}$

The conversion of the absorbed light to heat proceeds through three main photo-physical processes. The first and second processes allow for the formation of a hot metallic lattice in the metal NPs, and the last one allows for a cooling off, which means heat dissipation from the NPs to the surrounding environment. Accordingly, the dissipated energy will heat up the cancerous cells around the Au nanoparticles (AuNPs) causing cell destruction. However, such energy conversion and dissipation occur when the frequency of the incident light is overlapped with the SPR absorption band. ${ }^{24-27}$ Figure 2 shows the schematic presentation of the three processes included in the light to heat conversion mechanism.

On the other hand, exposure of the healthy cells to electromagnetic radiation of light raises the concern about the safety of this type of therapy. With respect to the LSPR frequency, the incident laser light is generally in the visible region or in the NIR region. In vivo, the radiation can be normally absorbed in the tissue by water, hemoglobin, and melanin. ${ }^{3}$ However, NIR laser light is weakly absorbed by these absorbents; hence, it can penetrate the skin without causing damage to normal tissues. ${ }^{24,28}$ Under such circumstances, many researchers have opened the door for the application of several nanomaterials, which strongly absorb the NIR and, as a result of SPR effect, generate sufficient heat to cause tumor cell death.

\section{Functional nanostructures as cancer PTT}

In this study, we introduce a recent literature review about nanomaterials used as a cancer PTT over the past 5 years. Different nanostructures developed even from the same material or combination of two or three PTT agents were reported to enhance the efficiency or to decrease the toxicity. For simplicity, this review covers mainly the application of diverse nano-scaled materials, either single or hybrid nanostructures, but does not cover the implication of any therapeutic agent or anticancer drug of the nanomaterials.

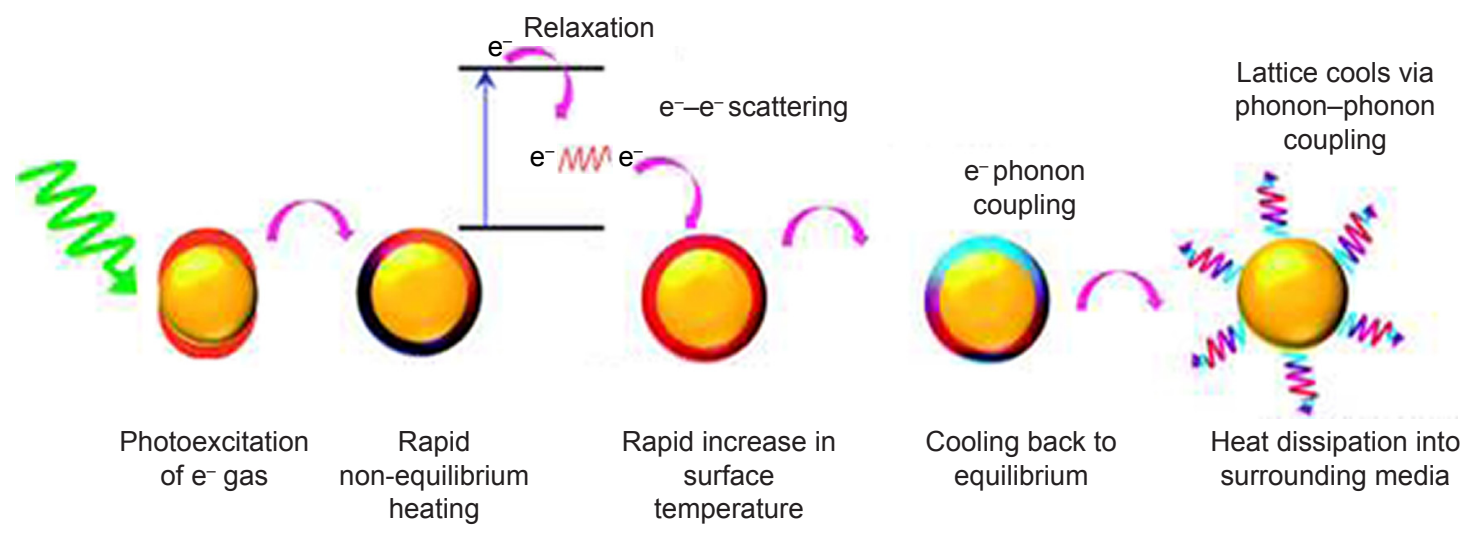

Figure 2 Schematic illustration of light to heat conversion by plasmonic nanostructures.

Notes: Step I is excitation of metal nanoparticles by the absorbed light photons, which results in particle oscillation and charge separation. Step 2 is conversion of the absorbed light to heat through electron-electron relaxation and electron-phonon relaxation processes, which result in the formation of hot metallic lattice. Step 3 is cooling off the metal structure through electron-phonon coupling and phonon-phonon relaxation, which cause heat dissipation. Reproduced from Webb JA, Bardhan R. Emerging advances in nanomedicine with engineered gold nanostructures. Nanoscale. 20I4;6(5):2502, ${ }^{27}$ http://pubs.rsc.org/en/content/articlelanding/20 I4/nr/c3nr05I I 2a\#!divAbstract, with permission of The Royal Society of Chemistry. 


\section{Au-based nanomaterials}

One of the most promising PTT nanomaterials for cancer treatment is Au nanostructures, as they can be synthesized in different forms such as nanorods, nanoshells, nanocages, or nanospheres. Beyond their plasmonic effect, they have an enhanced permeability to tumor vasculature, resulting in higher retention in tumor tissue. Because the tumor vascular system possesses gaps ranging in size from $100 \mathrm{~nm}$ to $2 \mu \mathrm{m}$, AuNPs can penetrate easily; these NPs are retained due to lymphatic clearance deficiency in the cancerous cells. ${ }^{18}$ Although AuNPs can successfully pass into the tumor tissue, they are subjected to phagocytosis by macrophages. Hence, AuNPs are sometimes coated with biocompatible polymers to inhibit reuptake by phagocytic cells..$^{29}$ In addition, AuNPs were reported as good biocompatible NPs with low cytotoxicity. ${ }^{30}$ Nanomaterials are unable to differentiate between cancerous cells and normal cells. In some studies, however, AuNPs were conjugated with a specific antibody-targeted cancer receptor to achieve minimal binding with healthy tissues. ${ }^{31-33}$

The optical properties of different Au nanostructures are greatly affected by altering their shapes and sizes. Research studies used these properties to tune the resonance in the NIR region according to the desired applications. ${ }^{27}$ In detail, more complex shapes such as Au nanorods (AuNRs), nanoshells, and nanostars exhibited more enhanced LSPR effect than simple AuNPs. ${ }^{34,35}$ Kelly et a ${ }^{36}$ demonstrated that complex geometrical shapes improved the polarizability of Au nanostructures due to additional bumps and protrusions. To illustrate, polarizability of AuNPs arises from their diameter only, while polarizability of Au-nanostar was enhanced by their projections, which increase the overall polarizability resulting in red shift in the plasmon resonance wavelength. ${ }^{37}$ In addition to shape, increasing the size of Au nanostructures enhances the electron oscillation around $\mathrm{Au}$ nanostructure leading to increasing the oscillation time and decreasing the frequency. This phenomenon is called phase retardation, which results in prolonged lifetime of plasmon and red shift of the resonance wavelength as well..$^{27,38}$

Findings from recent research support the application of Au-based nanomaterials as PTT. Ali et a ${ }^{39}$ studied the effect of AuNRs on human oral squamous cell carcinoma (HSC-3) by analyzing the data from Raman spectroscopy, metabolomics, and proteomics mass spectrometry. They claimed that the AuNR-treated cancer cells exhibited high levels of phenylalanine resulting in cell apoptosis. Similarly, a long-term in vivo study was carried out in mouse tumor tissues to discover the efficiency of AuNRs as PTT agents. ${ }^{40}$ The authors compared the apoptotic effect induced by gold nanorods conjugated to rifampicin (AuNRs@RF) to the apoptotic effect induced by gold nanorods conjugated to polyethelyne glycole (AuNRs@ PEG). By using proteomics analysis, it was found that AuNRs@ RF has more efficient endocytosis and easier cellular uptake resulting in increased neutrophil cell death (NETosis).

Moreover, AuNRs were found to minimize metastasis via inhibition of cancer cell migration. ${ }^{41}$ This occurs because AuNR surface, when conjugated to specific peptides, induces changes in migration-related proteins (integrins), which result in controlling cancer metastasis. Another key point is the effect of Au nanomaterial concentration on NIR-induced hyperthermia. Singh et al ${ }^{42}$ investigated the optimal concentration of AuNRs to be an efficient PTT in vitro using human esophagogastric tissues. They studied different concentrations of AuNRs and variable power densities. Their results showed a linear relationship between temperature elevation and laser power and AuNR concentration.

During treatment with a PTT agent, excessive reactive oxygen species (ROS) could be produced as a result of heat shock. ${ }^{43-45}$ Thus, ROS can cause protein damage leading to cell death. Sometimes, ROS levels increased in degenerative diseases such as Alzheimer or even in progressive disease such as cancer. ${ }^{46-49}$ Interestingly, platinum-coated Au nanorods were proved to be used in cancer therapy as a PTT with simultaneous ROS scavenging. ${ }^{50}$ Platinum shells of varying thickness were examined and showed no interference with the photothermal effect of AuNRs, as well as no impairment of the thermal response to NIR laser waves.

In addition, a new strategy was reported by $\mathrm{Wu}$ et al ${ }^{51}$ to synthesize nanotubes of Au-attapulgite composite, where attapulgite is a natural and biocompatible clay. The nanocomposite was prepared by the assembly of AuNPs on the surface of attapulgite. The authors stated that the synthesized tube-like nanocomposite could act as an efficient PTT when tested using A549 cells (human adenocarcinoma cell). Furthermore, a new Au nanostructure (Au nanobipyramids) was reported to be applied for breast cancer detection and treatment in which Au nano-bipyramids were bio-conjugated with 2-naphthalenethiol and folic acid to target and kill human breast (adenocarcinoma) cells. ${ }^{52}$ The authors revealed that the conjugated Au nano-bipyramids are capable of quantitative detection of the MCF-7 cells as well as enhancement of photothermal effect in vivo and in vitro.

On the contrary, there are some challenges in the clinical application of Au nanostructures as PTT agents. For instance, lack of stability inside the body and agglomeration of Au nanostructures is the most challenging factor upon in vivo application. ${ }^{27}$ Some modifications, such as attaching a 
surface ligand and dispersion in phosphate buffer with suitable $\mathrm{pH}$, could be carried out during synthesis to increase the in vivo stability and decrease the aggregation, respectively. ${ }^{53}$ However, the use of natural surface ligands results in lower yield from Au nanostructures, ${ }^{54}$ and the use of high salt or basic buffer can cause surface instability and aggregation of the nanostructures. ${ }^{55}$

\section{Iron oxide $\left(\mathrm{Fe}_{3} \mathrm{O}_{4}\right) \mathrm{NPs}$}

Due to unique magnetism, non-toxicity, biodegradability, and biocompatibility, magnetite $\mathrm{Fe}_{3} \mathrm{O}_{4} \mathrm{NPs}$ have been widely reported in biomedical applications. ${ }^{56-61}$ For instance, $\mathrm{Fe}_{3} \mathrm{O}_{4}$ NPs were extensively used in drug delivery systems, cell targeting, and in magnetic resonance imaging (MRI) as contrast agents. In addition, they possess a unique surface, which is able to carry targeting molecules for cancer cells and go deeply into tumor tissues. ${ }^{62}$ Furthermore, $\mathrm{Fe}_{3} \mathrm{O}_{4}$ NPs have been proved useful for cancer treatment due to their magnetic hyperthermia in which induced high temperatures were achieved and controlled by alternating magnetic field (AFM). ${ }^{63}$ The generated heat is sufficient to destroy cancerous cells but may affect healthy tissues. For this reason, the use of NIR laser radiation (widely used in therapy and diagnosis) to induce hyperthermia has an added safety advantage compared to AFM. ${ }^{64}$

$\mathrm{Fe}_{3} \mathrm{O}_{4}$ nanomaterials of different shapes (ie, spherical, hexagonal, and wire-like particles) were investigated for photothermal effect on human esophageal cancer cells (Eca-109). It was found that NIR-induced hyperthermia by spherical $\mathrm{Fe}_{3} \mathrm{O}_{4}$ NPs exhibited significant cytotoxicity and suppression of tumor growth. ${ }^{64}$ Shen et $\mathrm{al}^{65}$ have synthesized both individual and clustered $\mathrm{Fe}_{3} \mathrm{O}_{4}$ NPs and investigated their thermo-therapeutic effect in vivo and in vitro. They used human lung adenocarcinoma epithelial (A549) cells for in vitro testing and flow cytometry analysis to investigate the cell apoptosis effect. The clustered $\mathrm{Fe}_{3} \mathrm{O}_{4}$ NPs were shown to have higher NIR-induced hyperthermia and increased cell death compared to individual one.

The effect of coating $\mathrm{Fe}_{3} \mathrm{O}_{4}$ NPs with an anti-biofouling polymer was studied and compared to commercial magnetic NPs (FeREX, BioPAL) for their anticancer activity. ${ }^{66}$ The study showed that highly crystallized iron oxide nanoparticles (HCIONPs) offered more potential photothermal effect in vivo and in vitro due to their preferred lattice orientations. Consequently, this approach was suggested for clinical use in cancer treatment. Furthermore, Zhou et a ${ }^{67}$ developed iron/iron oxide core-shell nanoparticles $\left(\mathrm{Fe} @ \mathrm{Fe}_{3} \mathrm{O}_{4} \mathrm{NPs}\right)$ that have advantages of NIR photothermal therapeutic effect, selective magnetic targeting, and MRI for complete tumor treatment. Another study investigated the efficiency of FePt NPs functionalized with folates to target breast cancer cells EMT- $6 .{ }^{68}$ The findings revealed that the FePt NP exhibited intracellular explosions accompanied by rupture of cancerous cell membranes.

The main limitation of most studies is the use of highly intense laser irradiation, which might exceed the safe limit for cutaneous tissues $\left(0.33 \mathrm{~W} / \mathrm{cm}^{2}\right)$. However, Espinosa et $\mathrm{al}^{69}$ mitigated this drawback and reported the dual magnetic and photothermal activity of $\mathrm{Fe}_{3} \mathrm{O}_{4}$ nanocubes. The authors applied a magnetic field in alternating patterns and NIR laser of reasonable irradiation density $\left(0.3 \mathrm{~W} / \mathrm{cm}^{2}\right)$. Such dual approach showed complete cell death and tumor ablation even at low iron doses.

\section{Organic nanomaterials}

Owing to their enhanced biocompatibility and low toxicity, organic nanomaterials have recently been reported for a wide variety of biomedical applications. ${ }^{70}$ In particular, they can be used as PTT agents because they exhibit an efficient light-toheat conversion. Moreover, organic materials have a strong NIR absorption resulting from their diffused bi-electrons. ${ }^{71}$ Therefore, reduced graphene oxide (rGO) nanosheets as an example have a high extension coefficient $\left(3.6 \mathrm{~L} \mathrm{~g} \mathrm{~g}^{-1} \mathrm{~cm}^{-1}\right){ }^{72}$ Carbon-based nanomaterials (ie, carbon nanotubes and graphene), organic dyes, and polymer nanocarriers are the most widely reported organic-based photothermal nanoagents.

Liang et $\mathrm{al}^{73}$ have synthesized PEGylated single-walled carbon nanotubes (SWCNTs) and demonstrated their efficiency as a PTT. They investigated the therapeutic effect of PEGylated SWCNTs on metastasis of primary 4T1 murine breast tumor to lymph node. The findings showed the ability of PEGylated SWCNTs to suppress the primary tumor and the pulmonary metastasis. Similarly, a novel SWCNT-peptide composite showed an effective photothermal effect on colon 26 and HepG 2 cells after 3 minutes of NIR radiation. ${ }^{74}$

Graphene, as a carbon-based nanomaterial, has biomedical applications such as biosensors, drug carriers, and a very recent application as a PTT due to its strong NIR absorption. ${ }^{75-77}$ However, graphene is still considered to exhibit some biological toxicity because of poor dispersibility in physiological fluids. Consequently, most of the graphenebased PTT agents were modified with surface functionalization to mitigate the bio-toxicity limitation. ${ }^{77-79}$ To figure out photothermal efficiency of graphene and graphene oxide (GO), their ability to produce heat after laser irradiation was investigated and compared to AuNRs using an integrating sphere method and the time constant method. ${ }^{80}$

Graphene or GO as two-dimensional (2D) nanosheets with large surface area can be mixed with certain functional NPs for diagnostic and therapeutic purposes ${ }^{81}$ For example, 
$\mathrm{BaGdF}_{5} \mathrm{NPs}$ attached to the PEG-coated GO surface were fabricated by Zhang et $\mathrm{al}^{82}$ for both MRI and photothermal cancer therapy. The synthesized nanocomposites were injected into mice bearing HeLa tumor and irradiated for 10 minutes with $808 \mathrm{~nm}$ laser (power density of $0.5 \mathrm{~W} / \mathrm{cm}^{2}$ ). When the tumor tissue samples were collected for histological examination, the results showed that the treated group acquired thermonecrosis and cell shrinkage. Chen et $\mathrm{al}^{83}$ developed radiolabeled reduced GO coated with PEG ( $\left.{ }^{131} \mathrm{I}-\mathrm{rGO}-\mathrm{PEG}\right)$ for combined radiotherapy and PTT. The results showed high synergistic effect on 4T1 murine breast cancer therapy due to the combination between radioactive ${ }^{131} \mathrm{I}$ and strong NIR absorption of PEG-coated rGO as well as no apparent animal toxicity.

The literature revealed many articles concerned with the use of organic dyes such as cyanine and porphyrin derivatives as efficient photothermal agents. The most common cyanine derivative indocyanine green (ICG) dye, which was approved by the US Food and Drug Administration for clinical use, was reported in combination with nanocarriers as a PTT with a good efficiency in vitro and in vivo for tumor ablation. ${ }^{84-86}$ The nanocarriers containing ICG dye showed enhanced aqueous stability and better cellular uptake compared to the direct dyes. However, most of these studies have poor photostability at high-power laser irradiation, which means limited use to preclinical studies. ${ }^{70}$ Similarly, porphyrinbased nanomaterials showed its activity as PTT for the treatment of different tumors as well as their biodegradability and biocompatibility. ${ }^{87}$ However, it is important to mention that porphyrin nanoagents exhibit short absorption wavelength $(600-700 \mathrm{~nm})$, which restricts their in vivo application. ${ }^{70}$

In the last decade, NPs of conductive polymers have been reported for biomedical applications due to their high stability and biocompatibility. In particular, polypyrrole (PPy) and poly-(3,4-ethylenedioxythiophene):poly(4-styrenesulfonate) (PEDOT:PSS) have strong NIR absorbance, making them suitable as photothermal agents for cancer therapy. Both PPy and PEDOT:PSS were examined for tumor ablation in vivo and in vitro, showing high efficiency without undesired effects. ${ }^{88-91}$ However, the clinical use of polymer NPs is still limited due to lack of safety data. ${ }^{70}$

\section{Combined nanomaterials}

In general, the combination between two PTT agents to synthesize nanocomposites or core-shell NPs provides novel physical and chemical properties. ${ }^{92}$ In addition, these nanocomposites act as multi-function nanomaterials with tunable size and composition. The optical properties were enhanced while using two or more nanostructures to synthesize a hybrid PTT. ${ }^{93}$ Therefore, strong plasmonic effect and surface-enhanced Raman scattering were reported for many combined PTT.

Hybrid NPs consisting of $\mathrm{Au}$ and $\mathrm{Fe}_{3} \mathrm{O}_{4}$ were synthesized to get new and unique physical and chemical properties. The plasmonic effect was investigated by using optical absorption measurements such as UV-vis ${ }^{92}$ and surface-enhanced Raman spectra. ${ }^{93}$ Owing to their potential application as PTT, the optical properties of $\mathrm{Fe}_{3} \mathrm{O}_{4} @ \mathrm{Au}$ core-shell were simulated by using the extended Mie theory. ${ }^{94-96}$ Investigations of absorption cross-section spectra as well as the relationship between the resonance wavelength and the elevated temperature were conducted.$^{97}$ In addition, Ahmed et a ${ }^{98}$ studied the in vitro cell viability of $\mathrm{Fe}_{3} \mathrm{O}_{4} @$ AuNPs by using the MTT assay. They found $50 \mu \mathrm{g} / \mathrm{mL}$ of $\mathrm{Fe}_{3} \mathrm{O}_{4} @$ AuNPs to be non-toxic and biocompatible.

Another example of hybrid therapy is the combination between ICG organic dye and $\mathrm{Fe}_{3} \mathrm{O}_{4}$ NPs investigated by Niu et al. ${ }^{99}$ The authors used the two synergistic PTT agents encapsulated in perfluoropentane-based polylactide-co-glycolide ( $\mathrm{Fe}_{3} \mathrm{O}_{4} / \mathrm{ICG} @$ PLGA/PFP NPs) and evaluated their efficiency toward tumor ablation. When $\mathrm{Fe}_{3} \mathrm{O}_{4} / \mathrm{ICG} @$ PLGA/PFP NPs were incubated with MCF-7 human breast cancer cells and irradiated with $808 \mathrm{~nm}$ laser for 5 minutes, they showed tissue deformation and significant cell death. Furthermore, effectiveness of this dual NIR therapy was assessed in vivo by using mice bearing MCF-7 cells. The results revealed reduced tumor size and inhibition of tumor growth with no significant toxicity in vital organs such as liver, kidney, heart, spleen, and brain.

Meng et $\mathrm{al}^{100}$ developed golden SWCNT hybrids with enhanced NIR absorption, excellent water dispersibility, and better biocompatibility. The novel hybrids were synthesized by layer-by-layer self-assembly of two different polysaccharides on the SWCNT skeleton, followed by growing AuNPs on the surface. Highly effective photothermal activity was achieved, when the golden SWCNTs were incubated overnight with HeLa cells and irradiated for 15 minutes with $808 \mathrm{~nm}$ laser diode.

In addition, graphene-based hybrid PTT has been recently reported to have the advantages of carbon-based materials and metal NPs. For example, combination of GO with AuNPs revealed an enhanced tumor ablation in vivo and in vitro at lower laser irradiation $\left(0.75 \mathrm{~W} / \mathrm{cm}^{2}\right) .{ }^{101}$ Similarly, reduced $\mathrm{GO} / \mathrm{AuPd} \mathrm{NPs} / \mathrm{PEG}$-coated nanocomposites were evaluated for the suppression of HeLa cells in vitro showing good results after laser irradiation for 10 minutes. ${ }^{102}$ For the purpose of improving light-to-heat conversion as well as targeting the tumor tissues, combined $\mathrm{GO} /$ magnetic iron NP hybrids were also reported in the literature. ${ }^{81,103}$ However, 
laser-induced hyperthermia in these articles were substituted by magnetic hyperthermia, which is induced by AFM to mitigate the limited penetration of NIR into the tissues. ${ }^{104}$

Nanocomposites of AuNRs coated with pyrrole conductive polymer have gained a great interest due to high photostability and biocompatibility in addition to improved photothermal activity. ${ }^{105}$ Likewise, Au/pyrrole NPs with $\mathrm{Fe}_{3} \mathrm{O}_{4}$ NPs deposited on the surface were synthesized by Feng et $\mathrm{al}^{106}$ for cancer therapy. The Au/pyrrole@ $\mathrm{Fe}_{3} \mathrm{O}_{4}$ nanocomposites were considered to be multifunctional nanoagents with enhanced photothermal conversion, superparamagnetic properties, and good biocompatibility.

\section{Novel 2D materials}

New family of 2D nanosheets have been recently reported in the literature for many biomedical applications. Owing to high biocompatibility, excellent physicochemical performance as well as unique photothermal conversion efficiency, various 2D nanostructures are considered to be promising PTT agents for cancer therapy. ${ }^{107} \mathrm{MXenes}$ are recently reported but widely spread new 2D materials, having a general formula of $\mathrm{M}_{\mathrm{n}+1} \mathrm{X}_{\mathrm{n}}$, where $\mathrm{M}$ represents a transition metal and $\mathrm{X}$ represents carbon or nitrogen. ${ }^{108,109}$ One of the most powerful 2D PTT nanoagents is $\mathrm{Ti}_{3} \mathrm{C}_{2}$ (MXene), which shows strong NIR absorption and high light-to-heat conversion property. To explain, the exfoliated ultrathin MXene nanosheets demonstrate semimetal-like band energy structure; consequently, they have the LSPR effect similar to metal NPs. ${ }^{110}$ The same condition is applied for the transition of metal dichalcogenides such as tungsten sulfide $\left(\mathrm{WS}_{2}\right)$, molybdenum sulfide $\left(\mathrm{MoS}_{2}\right)$, and titanium sulfide $\left(\mathrm{TiS}_{2}\right)$ as well as black phosphorus. ${ }^{111,112}$

Lin et al ${ }^{110}$ synthesized phospholipid-stabilized ultrathin $\mathrm{Ti}_{3} \mathrm{C}_{2}$ nanosheets (MXene) by prolonged etching of $\mathrm{Ti}_{3} \mathrm{AlC}_{2}$ using hydrofluoric acid (HF), followed by intercalation with tetrapropylammonium hydroxide (TPAOH) and surface modification with soybean phospholipids (SP). They evaluated the cytotoxicity of $\mathrm{Ti}_{3} \mathrm{C}_{2}$-SP nanoflakes and the tumor ablation ability in vivo and in vitro by using 4T1 tumor-bearing mice and murine 4T1 breast cancer cells, respectively. The results showed that the groups treated with the stabilized MXenes revealed high percentage of 4T1 cancer cell death in vitro and significant decrease of tumor size in vivo after exposure to NIR laser irradiation for few minutes. Similarly, by using the same mouse model, MXene-based hybrids such as $\mathrm{MnO}_{\mathrm{x}}-\mathrm{Ti}_{3} \mathrm{C}_{2}$ composites were prepared and investigated for tumor ablation and MRI by Dai et al. ${ }^{113}$

Table 1 summarizes different nano-scaled PTT agents applied for different types of cancer cells. From Table 1, it is clearly observed that the combination between two or more PTT agents contributed in reducing the power densities of

Table I Summary of different nanomaterials reported as PTT agents applied on various types of tumor cells

\begin{tabular}{|c|c|c|c|}
\hline PTT nanoagent & Cancer cell type & Photothermal conditions & References \\
\hline \multicolumn{4}{|l|}{ Au-based nanomaterials } \\
\hline AuNRs & Human oral squamous cell carcinoma (HSC-3) & $\begin{array}{l}808 \mathrm{~nm} \text { NIR laser irradiation } \\
\left(5.8 \mathrm{~W} / \mathrm{cm}^{2}\right) \text { for } 3 \mathrm{~min}\end{array}$ & 41 \\
\hline Au-attapulgite composite & Human lung adenocarcinoma cells (A549) & $808 \mathrm{~nm}$ laser $\left(0.5 \mathrm{~W} / \mathrm{cm}^{2}\right)$ for $15 \mathrm{~min}$ & 51 \\
\hline AuNBPs & Human breast cancer cells (MCF-7) & $808 \mathrm{~nm}$ laser $\left(2 \mathrm{~W} / \mathrm{cm}^{2}\right)$ for $5 \mathrm{~min}$ & 52 \\
\hline \multicolumn{4}{|l|}{ Magnetic $\mathrm{Fe}_{3} \mathrm{O}_{4}$ nanomaterials } \\
\hline $\mathrm{Fe}_{3} \mathrm{O}_{4} \mathrm{NPs}$ & A549 cells & $808 \mathrm{NIR}\left(5 \mathrm{~W} / \mathrm{cm}^{2}\right)$ for $\mathrm{I}-3 \mathrm{~min}$ & 65 \\
\hline $\mathrm{Fe}_{3} \mathrm{O}_{4}$ nanocubes & Three malignant cell lines (SKOV3, PC3, and A43I) & $\begin{array}{l}\text { Dual magneto-photothermal } \\
\text { (alternating magnetic field and NIR } \\
\text { laser }\left[0.3 \mathrm{~W} / \mathrm{cm}^{2} \text { for } 5 \mathrm{~min}\right] \text { ) }\end{array}$ & 69 \\
\hline \multicolumn{4}{|l|}{ Organic nanomaterials } \\
\hline SWCNT composite & $\begin{array}{l}\text { The murine rectum carcinoma cell line (colon } 26) \text { and } \\
\text { human hepatocellular carcinoma cell line (HepG2) }\end{array}$ & $\begin{array}{l}808 \mathrm{~nm} \text { NIR laser }\left(1.2 \mathrm{~W} / \mathrm{cm}^{2}\right) \\
\text { for } 3 \mathrm{~min}\end{array}$ & 74 \\
\hline $\mathrm{GO}-\mathrm{BaGdF}_{5}$ nanocomposites & Mice bearing HeLa tumor cells & $808 \mathrm{~nm}$ laser $\left(0.5 \mathrm{~W} / \mathrm{cm}^{2}\right)$ for $10 \mathrm{~min}$ & 82 \\
\hline Polypyrrole hollow microspheres & Human umbilical vein endothelial cells (HUVECs) & $808 \mathrm{~nm}$ laser $\left(6 \mathrm{~W} / \mathrm{cm}^{2}\right)$ for $5 \mathrm{~min}$ & 90 \\
\hline \multicolumn{4}{|l|}{ Combination therapy } \\
\hline $\mathrm{Fe}_{3} \mathrm{O}_{4} / \mathrm{ICG} @ P L G A / P F P ~ N P s$ & MCF-7 & $808 \mathrm{~nm}$ laser for $5 \mathrm{~min}$ & 99 \\
\hline GO/AuNPs & Squamous cell carcinoma (SSC7) & $808 \mathrm{~nm}$ laser $\left(0.3 \mathrm{~W} / \mathrm{cm}^{2}\right)$ for $10 \mathrm{~min}$ & 101 \\
\hline AuPd NPs-rGO nanocomposites & HeLa cells & $808 \mathrm{~nm}$ laser $\left(\mathrm{I} \mathrm{W} / \mathrm{cm}^{2}\right)$ for $10 \mathrm{~min}$ & 102 \\
\hline \multicolumn{4}{|l|}{ Novel 2D materials } \\
\hline $\begin{array}{l}\mathrm{Ti}_{3} \mathrm{C}_{2} \text { nanosheets (MXenes) } \\
\text { Others }\end{array}$ & Murine 4TI breast cancer cells & 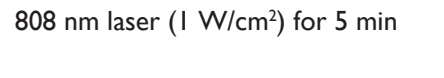 & 110 \\
\hline Antimony NPs & 4TI cells & $808 \mathrm{~nm}$ laser $\left(2 \mathrm{~W} / \mathrm{cm}^{2}\right)$ for $5 \mathrm{~min}$ & 114 \\
\hline Iron carbide $\left(\mathrm{Fe}_{5} \mathrm{C}_{2}\right) \mathrm{NPs}$ & Human ovarian cancer cell line (SK-OV-3) & $808 \mathrm{~nm}$ laser $\left(0.8 \mathrm{~W} / \mathrm{cm}^{2}\right)$ for $5 \mathrm{~min}$ & 115 \\
\hline Tungsten oxide nanorods & HeLa cells & $980 \mathrm{~nm}$ laser $\left(0.35 \mathrm{~W} / \mathrm{cm}^{2}\right)$ for $8 \mathrm{~min}$ & 116 \\
\hline
\end{tabular}

Abbreviations: AuNRs, gold nanorods; AuNBPs, gold nanobipyramids; GO, graphene oxide; NIR, near-infrared; NP, nanoparticle; PTT, photothermal therapy; SWCNT, single-walled carbon nanotube. 
laser irradiation near to the safety limit in addition to decreasing the exposure radiation time.

\section{Conclusion and future perspective}

Throughout this review, new insights for cancer treatment have been addressed, focusing on the promising benefits of employing the PTT types. It is evident from ineffective conventional treatments that there is a desperate necessity for distinct and unique treatments. As extensively explained, nanostructure technology has the potential to play critical functions in cancer therapy, through directly acting as PTT. Of note, nanostructures with various shapes were revealed as PTT due to their strong NIR absorption and efficient light-to-heat conversion. Investigation through various nanostructure studies revealed that the mentioned concepts attempt to strengthen their overall photothermal therapeutic behavior. As represented by numerous reports, therapies including AuNPs, $\mathrm{Fe}_{3} \mathrm{O}_{4} \mathrm{NPs}$, organic nanomaterials, and novel 2D nanoagents have the potential to directly enhance the in vivo and in vitro cancer therapies. In addition, hybrid nanomaterials with two or more PTT agents have superior advantages of synergism, which leads to enhanced efficiency and reduced toxicological effects of every single therapy. To sum up, nanostructures are considered promising candidates for tumor ablation as PTT agents due to their unique optical properties. However, in vivo stability and more detailed toxicity studies should be covered in the future for the PTT nanoagents to be clinically approved.

\section{Acknowledgments}

The authors gratefully acknowledge Qatar University for Graduate Assistantship and they would also like to show gratitude to Dr Mary Newsome (Office of Graduate Studies, Qatar University) for discussions and useful suggestions of writing this paper. The authors would also like to acknowledge Qatar University for funding the project: GCC Co-Fund Program Grant GCC-2017-001.

\section{Disclosure}

The authors report no conflicts of interest in this work.

\section{References}

1. Siegel RL, Miller KD, Jemal A. Cancer statistics, 2017. CA Cancer J Clin. 2017;67(1):7-30.

2. Cancer Fact Sheet. World Health Organization; 2017. Available from: http://www.who.int/mediacentre/factsheets/fs297/en/. Accessed December $12,2017$.

3. Day ES, Morton JG, West JL. Nanoparticles for thermal cancer therapy. J Biomech Eng. 2009;131(7):74001.

4. Melancon MP, Zhou M, Li C. Cancer theranostics with near-infrared light-activatable multimodal nanoparticles. Acc Chem Res. 2011;44(10): 947-956.
5. Kennedy LC, Bickford LR, Lewinski NA, et al. A new era for cancer treatment: Gold-nanoparticle-mediated thermal therapies. Small. 2011;7(2):169-183.

6. Yang K, Feng L, Shi X, Liu Z. Nano-graphene in biomedicine: theranostic applications. Chem Soc Rev. 2013;42(2):530-547.

7. Camerin M, Rello S, Villanueva A, et al. Photothermal sensitisation as a novel therapeutic approach for tumours: studies at the cellular and animal level. Eur J Cancer. 2005;41(8):1203-1212.

8. Camerin M, Rodgers MAJ, Kenney ME, Jori G. Photothermal sensitization: evidence for the lack of oxygen effect on the photosensitizing activity. Photochem Photobiol Sci. 2005;4(3):251-253.

9. He X, Bischof JC. Quantification of temperature and injury response in thermal therapy and cryosurgery. Crit Rev Biomed Eng. 2003;31(5-6): 355-422.

10. van der Zee J. Heating the patient: a promising approach? Ann Oncol. 2002;13(8):1173-1184.

11. El-Sayed MA. Some interesting properties of metals confined in time and nanometer space of different shapes. Acc Chem Res. 2001;34(4):257-264.

12. Niemeyer CM. Nanoparticles, proteins, and nucleic acids: biotechnology meets materials science. Angew Chem . 2001;40(22):4128-4158.

13. West JL, Halas NJ. Engineered nanomaterials for biophotonics applications: improving sensing, imaging, and therapeutics. Annu Rev Biomed Eng. 2003;5(1):285-292.

14. Katz E, Willner I. Integrated nanoparticle-biomolecule hybrid systems: synthesis, properties, and applications. Angew Chem. 2004;43(45):6042-6108.

15. Dreaden EC, Alkilany AM, Huang X, Murphy CJ, El-Sayed MA. The golden age: gold nanoparticles for biomedicine. Chem Soc Rev. 2012; 41(7):2740-2779.

16. Dreaden EC, Mackey MA, Huang X, Kang B, El-Sayed MA. Beating cancer in multiple ways using nanogold. Chem Soc Rev. 2011;40(7):3391.

17. Huang X, Neretina S, El-Sayed MA. Gold nanorods: from synthesis and properties to biological and biomedical applications. Adv Mater. 2009;21(48):4880-4910.

18. Lee J, Chatterjee DK, Lee MH, Krishnan S. Gold nanoparticles in breast cancer treatment: promise and potential pitfalls. Cancer Lett. 2014; 347(1):46-53.

19. Jain PK, Huang X, El-Sayed IH, El-Sayed MA. Noble metals on the nanoscale: optical and photothermal properties and some applications in imaging, sensing, biology, and medicine. Acc Chem Res. 2008;41(12): $1578-1586$.

20. Huang X, Jain PK, El-Sayed IH, El-Sayed MA. Gold nanoparticles: interesting optical properties and recent applications in cancer diagnostics and therapy. Nanomedicine. 2007;2(5):681-693.

21. Huang X, Jain PK, El-Sayed IH, El-Sayed MA. Plasmonic photothermal therapy (PPTT) using gold nanoparticles. Lasers Med Sci. 2008;23(3): 217-228.

22. Jain PK, Lee KS, El-Sayed IH, El-Sayed MA. Calculated absorption and scattering properties of gold nanoparticles of different size, shape, and composition: applications in biological imaging and biomedicine. J Phys Chem B. 2006;110(14):7238-7248.

23. Huang X, El-Sayed MA. Gold nanoparticles: optical properties and implementations in cancer diagnosis and photothermal therapy. $J A d v$ Res. 2010;1(1):13-28.

24. Huang X, El-Sayed MA. Plasmonic photo-thermal therapy (PPTT). Alexandria J Med. 2011;47(1):1-9.

25. Xie T, Jing C, Long YT. Single plasmonic nanoparticles as ultrasensitive sensors. Analyst. 2017;142(3):409-420.

26. Link S, El-Sayed MA. Optical properties and ultrafast dynamics of metallic nanocrystals. Annu Rev Phys Chem. 2003;54(1):331-366.

27. Webb JA, Bardhan R. Emerging advances in nanomedicine with engineered gold nanostructures. Nanoscale. 2014;6(5):2502.

28. Kim J, Park S, Lee JE, et al. Designed fabrication of multifunctional magnetic gold nanoshells and their application to magnetic resonance imaging and photothermal therapy. Angew Chem. 2006;118(46): 7918-7922. 
29. Kah JCY, Wong KY, Neoh KG, et al. Critical parameters in the pegylation of gold nanoshells for biomedical applications: an in vitro macrophage study. J Drug Target. 2009;17(3):181-193.

30. Hu M, Chen J, Li Z-Y, et al. Gold nanostructures: engineering their plasmonic properties for biomedical applications. Chem Soc Rev. 2006; 35(11):1084-1094.

31. Lowery AR, Gobin AM, Day ES, Halas NJ, West JL. Immunonanoshells for targeted photothermal ablation of tumor cells. Int J Nanomedicine. 2006;1(2):149-154.

32. Wu P-C, Shieh D-B, Cheng F-Y. Nanomaterial-mediated photothermal cancer treatment: the pivotal role of cellular uptake on photothermal therapeutic efficacy. RSC Adv. 2014;4(95):53297-53306.

33. Loo C, Lowery A, Halas N, West J, Drezek R. Immunotargeted nanoshells for integrated cancer imaging and therapy. Nano Lett. 2005; 5(4):709-711.

34. Yuan H, Khoury CG, Wilson CM, Grant GA, Bennett AJ, Vo-Dinh T. In vivo particle tracking and photothermal ablation using plasmonresonant gold nanostars. Nanomedicine. 2012;8(8):1355-1363.

35. Ye X, Gao Y, Chen J, Relfsnyder DC, Zheng C, Murray CB. Seeded growth of monodisperse gold nanorods using bromide-free surfactant mixtures. Nano Lett. 2013;13(5):2163-2171.

36. Kelly KL, Coronado E, Zhao LL, Schatz GC. The optical properties of metal nanoparticles: the influence of size, shape, and dielectric environment. J Phys Chem B. 2003;107(3):668-677.

37. Burda C, Chen X, Narayanan R, El-Sayed MA. Chemistry and properties of nanocrystals of different shapes. Chem Rev. 2005;105(4):1025-1102.

38. Ringe E, Langille MR, Sohn K, et al. Plasmon length: a universal parameter to describe size effects in gold nanoparticles. J Phys Chem Lett. 2012;3(11):1479-1483.

39. Ali MRK, Wu Y, Han T, et al. Simultaneous time-dependent surfaceenhanced Raman spectroscopy, metabolomics, and proteomics reveal cancer cell death mechanisms associated with gold nanorod photothermal therapy. J Am Chem Soc. 2016;138(47):15434-15442.

40. Ali MRK, Rahman MA, Wu Y, etal. Efficacy, long-term toxicity, and mechanistic studies of gold nanorods photothermal therapy of cancer in xenograft mice. Proc Natl Acad Sci U S A. 2017;114(15):E3110-E3118.

41. Ali MRK, Wu Y, Tang Y, et al. Targeting cancer cell integrins using gold nanorods in photothermal therapy inhibits migration through affecting cytoskeletal proteins. Proc Natl Acad Sci US A. 2017;114(28): E5655-E5663.

42. Singh M, Harris-Birtill DCC, Zhou Y, et al. Application of gold nanorods for photothermal therapy in ex vivo human oesophagogastric adenocarcinoma. J Biomed Nanotechnol. 2016;12(3):481-490.

43. Slimen IB, Najar T, Ghram A, Dabbebi H, Ben Mrad M, Abdrabbah M. Reactive oxygen species, heat stress and oxidative-induced mitochondrial damage. A review. Int J Hyperth. 2014;30(7):513-523.

44. Fedyaeva AV, Stepanov AV, Lyubushkina IV, Pobezhimova TP, Rikhvanov EG. Heat shock induces production of reactive oxygen species and increases inner mitochondrial membrane potential in winter wheat cells. Biochem. 2014;79(11):1202-1210.

45. Gao L, Liu R, Gao F, Wang Y, Jiang X, Gao X. Plasmon-mediated generation of reactive oxygen species from near-infrared light excited gold nanocages for photodynamic therapy in vitro. ACS Nano. 2014;8(7): 7260-7271.

46. Benz CC, Yau C. Ageing, oxidative stress and cancer: paradigms in parallax. Nat Rev Cancer. 2009;8(11):875-879.

47. Uttara B, Singh A, Zamboni P, Mahajan R. Oxidative stress and neurodegenerative diseases: a review of upstream and downstream antioxidant therapeutic options. Curr Neuropharmacol. 2009;7(1):65-74.

48. Schieber M, Chandel NS. ROS function in redox signaling and oxidative stress. Curr Biol. 2014;24(10):R453-R462.

49. Cervellati F, Cervellati C, Romani A, et al. Hypoxia induces cell damage via oxidative stress in retinal epithelial cells. Free Radic Res. 2014;48(3):303-312.

50. Aioub M, Panikkanvalappil SR, El-Sayed MA. Platinum-coated gold nanorods: efficient reactive oxygen scavengers that prevent oxidative damage toward healthy, untreated cells during plasmonic photothermal therapy. ACS Nano. 2017;11(1):579-586.
51. Wu P, Deng D, Gao J, Cai C. Tube-like gold sphere-attapulgite nanocomposites with a high photothermal conversion ability in the nearinfrared region for enhanced cancer photothermal therapy. ACS Appl Mater Interfaces. 2016;8(16):10243-10252.

52. Feng J, Chen L, Xia Y, et al. Bioconjugation of gold nanobipyramids for SERS detection and targeted photothermal therapy in breast cancer. ACS Biomater Sci Eng. 2017;3(4):608-618.

53. Niidome T, Yamagata M, Okamoto Y, et al. PEG-modified gold nanorods with a stealth character for in vivo applications. J Control Release. 2006;114(3):343-347.

54. Ma Y, Chechik V. Aging of gold nanoparticles: ligand exchange with disulfides. Langmuir. 2011;27(23):14432-14437.

55. Wang Z, Lee JH, Lu Y. Label-free colorimetric detection of lead ions with a nanomolar detection limit and tunable dynamic range by using gold nanoparticles and DNAzyme. Adv Mater. 2008;20(17): 3263-3267.

56. Laurent S, Mahmoudi M. Superparamagnetic iron oxide nanoparticles: promises for diagnosis and treatment of cancer. Int J Mol Epidemiol Genet. 2011;2(4):367-390.

57. Maier-Hauff K, Ulrich F, Nestler D, et al. Efficacy and safety of intratumoral thermotherapy using magnetic iron-oxide nanoparticles combined with external beam radiotherapy on patients with recurrent glioblastoma multiforme. J Neurooncol. 2011;103(2):317-324.

58. Anselmo AC, Mitragotri S. A review of clinical translation of inorganic nanoparticles. AAPS J. 2015;17(5):1041-1054.

59. Lartigue L, Alloyeau D, Kolosnjaj-Tabi J, et al. Biodegradation of iron oxide nanocubes: high-resolution in situ monitoring. ACS Nano. 2013;7(5):3939-3952.

60. Lee J-H, Jang J, Choi J, et al. Exchange-coupled magnetic nanoparticles for efficient heat induction. Nat Nanotechnol. 2011;6(7):418-422.

61. Yang HW, Hua MY, Liu HL, et al. Self-protecting core-shell magnetic nanoparticles for targeted, traceable, long half-life delivery of BCNU to gliomas. Biomaterials. 2011;32(27):6523-6532.

62. Sohail A, Ahmad Z, Bég OA, Arshad S, Sherin L. A review on hyperthermia via nanoparticle-mediated therapy. Bull Cancer. 2017;104(5): $452-461$.

63. Rosensweig RE. Heating magnetic fluid with alternating magnetic field. J Magn Magn Mater. 2002;252(1-3 SPEC. ISS.):370-374.

64. Chu M, Shao Y, Peng J, et al. Near-infrared laser light mediated cancer therapy by photothermal effect of Fe3O4magnetic nanoparticles. Biomaterials. 2013;34(16):4078-4088.

65. Shen S, Wang S, Zheng R, et al. Magnetic nanoparticle clusters for photothermal therapy with near-infrared irradiation. Biomaterials. 2015;39:67-74.

66. Chen H, Burnett J, Zhang F, Zhang J, Paholak H, Sun D. Highly crystallized iron oxide nanoparticles as effective and biodegradable mediators for photothermal cancer therapy. J Mater Chem B. 2014;2(7):757-765.

67. Zhou Z, Sun Y, Shen J, et al. Iron/iron oxide core/shell nanoparticles for magnetic targeting MRI and near-infrared photothermal therapy. Biomaterials. 2014;35(26):7470-7478.

68. Chen CL, Kuo LR, Lee SY, et al. Photothermal cancer therapy via femtosecond-laser-excited FePt nanoparticles. Biomaterials. 2013; 34(4):1128-1134.

69. Espinosa A, Di Corato R, Kolosnjaj-Tabi J, Flaud P, Pellegrino T, Wilhelm C. Duality of iron oxide nanoparticles in cancer therapy: amplification of heating efficiency by magnetic hyperthermia and photothermal bimodal treatment. ACS Nano. 2016;10(2):2436-2446.

70. Wang J, Qiu J. A review of organic nanomaterials in photothermal cancer therapy. Cancer Res Front. 2016;2(1):67-84.

71. Yang $\mathrm{K}, \mathrm{Xu} \mathrm{H}$, Cheng L, Sun $\mathrm{C}$, Wang J, Liu Z. In vitro and in vivo near-infrared photothermal therapy of cancer using polypyrrole organic nanoparticles. Adv Mater. 2012;24(41):5586-5592.

72. Robinson JT, Tabakman SM, Liang Y, et al. Ultrasmall reduced graphene oxide with high near-infrared absorbance for photothermal therapy. J Am Chem Soc. 2011;133(17):6825-6831.

73. Liang C, Diao S, Wang C, et al. Tumor metastasis inhibition by imagingguided photothermal therapy with single-walled carbon nanotubes. Adv Mater. 2014;26(32):5646-5652. 
74. Hashida Y, Tanaka H, Zhou S, et al. Photothermal ablation of tumor cells using a single-walled carbon nanotube-peptide composite. J Control Release. 2014;173(1):58-66.

75. Jin Y, Wang J, Ke H, Wang S, Dai Z. Graphene oxide modified PLA microcapsules containing gold nanoparticles for ultrasonic/CT bimodal imaging guided photothermal tumor therapy. Biomaterials. 2013;34(20):4794-4802

76. Chen YW, Chen PJ, Hu SH, Chen IW, Chen SY. NIR-triggered synergic photo-chemothermal therapy delivered by reduced graphene oxide/carbon/mesoporous silica nanocookies. Adv Funct Mater. 2014; 24(4):451-459.

77. Gonçalves G. Graphene-Based Materials in Health and Environment. Vila GGPMM, editor. New York: Springer; 2016.

78. Bussy C, Ali-Boucetta H, Kostarelos K. Safety considerations for graphene: lessons learnt from carbon nanotubes. Acc Chem Res. 2013; 46(3):692-701.

79. Kostarelos K, Novoselov KS. Exploring the interface of graphene and biology. Science. 2014;344(6181):261-263.

80. Savchuk OA, Carvajal JJ, Massons J, Aguiló M, Díaz F. Determination of photothermal conversion efficiency of graphene and graphene oxide through an integrating sphere method. Carbon. 2016;103: 134-141.

81. Yang K, Hu L, Ma X, et al. Multimodal imaging guided photothermal therapy using functionalized graphene nanosheets anchored with magnetic nanoparticles. Adv Mater. 2012;24(14):1868-1872.

82. Zhang $\mathrm{H}$, Wu H, Wang J, et al. Graphene oxide-BaGdF5 nanocomposites for multi-modal imaging and photothermal therapy. Biomaterials. 2015;42:66-77.

83. Chen L, Zhong X, Yi X, et al. Radionuclide ${ }^{131}$ I labeled reduced graphene oxide for nuclear imaging guided combined radio- and photothermal therapy of cancer. Biomaterials. 2015;66:21-28.

84. Gong H, Dong Z, Liu Y, et al. Engineering of multifunctional nano-micelles for combined photothermal and photodynamic therapy under the guidance of multimodal imaging. Adv Funct Mater. 2014;24:6492-6502.

85. Jian W, Yu T, Chen C, et al. Indocyanine green-encapsulated hybrid polymeric nanomicelles for photothermal cancer therapy. Langmuir. 2015;31:6202-6210.

86. Kumar P, Srivastava R. IR 820 dye encapsulated in polycaprolactone glycol chitosan: Poloxamer blend nanoparticles for photo immunotherapy for breast cancer. Mater Sci Eng C. 2015;57:321-327.

87. Jin CS, Lovell JF, Chen J, Zheng G. Ablation of hypoxic tumors with not photodynamic, therapy using a nanostructured porphyrin assembly. ACS Nano. 2013;7(3):2541-2550.

88. Cheng L, Yang K, Chen Q, Liu Z. Organic stealth nanoparticles for highly effective in vivo near-infrared photothermal therapy of cancer. ACS Nano. 2012;6(6):5605-5613.

89. Chen M, Fang X, Tang S, Zheng N. Polypyrrole nanoparticles for high-performance in vivo near-infrared photothermal cancer therapy. Chem Commun. 2012;48(71):8934.

90. Zha Z, Wang J, Qu E, et al. Polypyrrole hollow microspheres as echogenic photothermal agent for ultrasound imaging guided tumor ablation. Sci Rep. 2013;3:1-8.

91. Liang X, Li Y, Li X, et al. PEGylated polypyrrole nanoparticles conjugating gadolinium chelates for dual-modal MRI/photoacoustic imaging guided photothermal therapy of cancer. Adv Funct Mater. 2015;25:1451-1462.

92. Yu H, Chen M, Rice PM, Wang SX, White RL, Sun S. Dumbbell-like bifunctional Au-Fe3O4 nanoparticles. Nano Lett. 2005;5(2):379-382.

93. Mezni A, Balti I, Mlayah A, et al. Hybrid Au-Fe3O4 nanoparticles: Plasmonic, surface enhanced Raman scattering, and phase transition properties. J Phys Chem C. 2013;117(31):16166-16174.

94. Brullot W, Valev VK, Verbiest T. Magnetic-plasmonic nanoparticles for the life sciences: calculated optical properties of hybrid structures. Nanomedicine. 2012;8(5):559-568.

95. Chaffin EA, Bhana S, O'Connor RT, Huang X, Wang Y. Impact of core dielectric properties on the localized surface plasmonic spectra of gold-coated magnetic core-shell nanoparticles. J Phys Chem B. 2014;118(49):14076-14084.
96. Li Q, Zhang W, Zhao D, Qiu M. Photothermal enhancement in coreshell structured plasmonic nanoparticles. Plasmonics. 2014;9(3): 623-630.

97. Encina ER, Coronado EA. Size optimization of iron oxide@noble metal core-shell nanohybrids for photothermal applications. $J$ Phys Chem C. 2016;120(10):5630-5639.

98. Ahmed OS, Mohamed MB, Zekri ARN, Imam H, Khaled HM, Abdel-kader MH. Synthesis of the newly developed core-shell Au/ Fe3O4 magnato-plasmonic nanocomposite in cancer cells. Life Sci J. 2014;11(10):182-187.

99. Niu C, Xu Y, An S, et al. Near-infrared induced phase-shifted ICG/ Fe3O4 loaded PLGA nanoparticles for photothermal tumor ablation. Sci Rep. 2017;7(1):1-10.

100. Meng L, Xia W, Liu L, Niu L, Lu Q. Golden single-walled carbon nanotubes prepared using double layer polysaccharides bridge for photothermal therapy. ACS Appl Mater Interfaces. 2014;6(7):4989-4996.

101. Gao S, Zhang L, Wang G, et al. Hybrid graphene/Au activatable theranostic agent for multimodalities imaging guided enhanced photothermal therapy. Biomaterials. 2016;79:36-45.

102. Darabdhara G, Das MR, Turcheniuk V. Reduced graphene oxide nanosheets decorated with AuPd bimetallic nanoparticles: a multifunctional material for photothermal therapy of cancer cells. J Mater Chem B. 2015;3(42):8366-8374.

103. Akhavan O, Meidanchi A, Khoei S. Zinc ferrite spinel-graphene in magneto-photothermal therapy of cancer. J Mater Chem B. 2014;2(21): 3306-3314.

104. Criado A, Prato M. Recent advances of graphene-based hybrids with magnetic nanoparticles for biomedical applications. Curr Med Chem. 2017;24(5):529-536

105. Du C, Wang A, Fei J, Zhao J, Li J. Polypyrrole-stabilized gold nanorods with enhanced photothermal effect towards two-photon photothermal therapy. J Mater Chem B. 2015;3(22):4539-4545.

106. Feng W, Zhou X, Nie W, et al. Au/polypyrrole@Fe3O4 nanocomposites for MR/CT dual-modal imaging guided-photothermal therapy: an in vitro study. ACS Appl Mater Interfaces. 2015;7(7):4354-4367.

107. Chen Y, Wang L, Shi J. Two-dimensional non-carbonaceous materialsenabled efficient photothermal cancer therapy. Nano Today. 2016; 11(3):292-308

108. Naguib M, Kurtoglu M, Presser V, et al. Two-dimensional nanocrystals produced by exfoliation of Ti 3AlC 2. Adv Mater. 2011;23(37): 4248-4253.

109. Karlsson LH, Birch J, Halim J, Barsoum MW, Persson PO. Atomically resolved structural and chemical investigation of single MXene sheets. Nano Lett. 2015;15(8):4955-4960.

110. Lin H, Wang X, Yu L, Chen Y, Shi J. Two-dimensional ultrathin MXene ceramic nanosheets for photothermal conversion. Nano Lett. 2017;17(1):384-391.

111. Cheng L, Liu J, Gu X, et al. PEGylated WS2 nanosheets as a multifunctional theranostic agent for in vivo dual-modal CT/photoacoustic imaging guided photothermal therapy. Adv Mater. 2014;26(12):1886-1893.

112. Zhu Z, Zou Y, Hu W, et al. Near-infrared plasmonic 2D semimetals for applications in communication and biology. Adv Funct Mater. 2016; 26(11):1793-1802.

113. Dai C, Lin H, Xu G, Liu Z, Wu R, Chen Y. Biocompatible 2D titanium carbide (MXenes) composite nanosheets for $\mathrm{pH}$-responsive MRIguided tumor hyperthermia. Chem Mater. 2017;29(20):8637-8652.

114. Li W, Rong P, Yang K, Huang P, Sun K, Chen X. Semimetal nanomaterials of antimony as highly efficient agent for photoacoustic imaging and photothermal therapy. Biomaterials. 2015;45:18-26.

115. Yu J, Ju Y, Zhao L, et al. Multistimuli-regulated photochemothermal cancer therapy remotely controlled via Fe5C2nanoparticles. ACS Nano. 2016;10(1):159-169.

116. Zhou Z, Kong B, Yu C, et al. Tungsten oxide nanorods: An efficient nanoplatform for tumor CT imaging and photothermal therapy. Sci Rep. 2014;4:1-10. 


\section{Publish your work in this journal}

The International Journal of Nanomedicine is an international, peerreviewed journal focusing on the application of nanotechnology in diagnostics, therapeutics, and drug delivery systems throughout the biomedical field. This journal is indexed on PubMed Central, MedLine, CAS, SciSearch $\AA$, Current Contents ${ }^{\circledR} /$ Clinical Medicine,

Journal Citation Reports/Science Edition, EMBase, Scopus and the Elsevier Bibliographic databases. The manuscript management system is completely online and includes a very quick and fair peer-review system, which is all easy to use. Visit http://www.dovepress.com/ testimonials.php to read real quotes from published authors.

Submit your manuscript here: http://www.dovepress.com/international-journal-of-nanomedicine-journal 